“ (C) 2018 IEEE. Personal use of this material is permitted. Permission from IEEE must be obtained for all other uses, in any current or future media, including

reprinting/republishing this material for advertising or promotional purposes, creating new collective works, for resale or redistribution to servers or lists, or reuse of any copyrighted component of this work in other works." 


\title{
Towards Carbon Based Artificial Impedance Surfaces for Conformal Aerospace Applications
}

\author{
A. Todd ${ }^{\# 1}$, T. C. Baum*, K. J. Nicholson*, R. W. Ziolkowski!'@ and K. Ghorbani ${ }^{\# 2}$

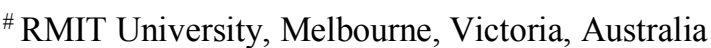 \\ * Defence Science and Technology Group, Melbourne, Victoria, Australia \\ ! University of Technology Sydney, Global Big Data Technologies Centre, Ultimo, NSW, Australia \\ @ University of Arizona, Department of Electrical and Computer Engineering, Tucson, AZ, USA \\ 1'alice.todd@rmit.edu.au, ${ }^{2}$ kamran.ghorbani@rmit.edu.au
}

\begin{abstract}
Conformal load-bearing smart skins (CLSS) provide the unique ability to integrate complex electrical systems into aerospace composite materials. This paper provides a preliminary investigation into the patterning of Artificial Impedance Surfaces (AIS) on these materials as they are well suited to develop conformal meta-surfaces (MTS). In particular, a composite veil material has been patterned using laser ablation and has been transferred directly onto a resin pre-impregnated (pre-preg) structural glass material. The new patterning technique was found suitable for the creation of fine geometric features with a resolution of approximately $100 \mu \mathrm{m}$. The material has been characterized by a reflected magnitude and phase measurement.

Keywords - ceramics, delay filters, power amplifiers, coaxial resonators.
\end{abstract}

\section{INTRODUCTION}

Future aerospace platforms demand better fuel economy, lighter weight and low drag to deliver high performance systems for customers. However, antennas and integrated transceivers, which form an integral part of modern aerospace platforms, stand-out as an area where significant innovation is possible. Traditionally, communication or radar sensors (incorporated in aerospace platforms) have been regarded as individual sub-systems. This is because the systems integrator (i.e. the company who assembles the platform) typically purchases these antennas and transceivers from separate original equipment manufacturers (OEM). Hence, the position of the antenna and transceiver is decided during the design phase of a platform. There is very little synergy in optimizing the electromagnetic and structural requirements.

Holographic meta-surfaces (MTS) are a promising technology to enable the development of large planar apertures [1] - [4]. This highly active research field in electromagnetics has provided new avenues to design conventional arrays in a more simplistic and novel manner. MTS are also an attractive solution for large scale aerospace conformal arrays and can, in theory, provide a much simpler means for integrating apertures directly into load-bearing structure. This type of antenna is classically referred to as a conformal load-bearing antenna structure (CLAS) [6] - [9] which falls under a recent avenue of synergistic design, referred to as multi-functional conformal load-bearing smart skins (CLSS) [10]. CLSS structures enable the direct manufacture of full RF transceivers directly in the skin of load-bearing structure with little degradation in the mechanical performance. CLSS would also remove the need for bulky, drag inducing radomes [5] and open the possibility for direct integration of active components for electronic beam steering [10], [12].

To enable CLSS, a carbon-based veil material has been previously proposed by the authors [8]-[9], [11]. This material is manufactured by TFP and is available commercially in a variety of different densities and conductivities. For many electromagnetic applications, the conductivity of carbon fiber is relatively poor [13]. However, veil materials can be plated in various metals to increase their conductivity to acceptable levels [11]. Veils are attractive as conductors for CLSS applications as they show little degradation in their conductivity performance under mechanical load. They also do not cause significant degradation in the mechanical performance of composites, unlike solid metal foils [8]. Combining the properties of the veil with the drapability of composite resin pre-impregnated (pre-preg) fabrics enables the development of conformal, holographic MTS [14]. To date, the majority of research into holographic MTS antennas has focused on planar or semi-conformal applications [1] - [5], [15]. With advancements in manufacturing and the availability of commercial electromagnetic simulation software, it is now possible to start investigating how to pattern these MTS structures onto truly conformal shapes.

This paper investigates a new patterning technique for carbon veils which enables direct transfer onto composite prepreg materials. It will be shown that this process produces superior resolution (down to $100 \mu \mathrm{m}$ ), which is well suited for patterning holographic artificial impedance surfaces (AIS). The technique is also robust and well suited for large scale manufacturing. Finally, it provides a unique avenue to develop truly conformal AIS.

\section{CARbon Veil And Patterning TeChnique}

Carbon fiber veils have traditionally been used in composite manufacturing as a cosmetic finishing for structural components. These materials also offer a unique solution to develop CLSS structures as they are highly porous, light weight 
and conductive. The chopped-strand carbon veils (supplied by TFP) used in this report are based on randomly orientated chopped-strand carbon fibers which exhibit low conductivity ( 28,000 S/m@10 GHz [12]). However, they can be plated in highly conductive metals such as copper. This composite conductor offers a number of advantages in structural applications. For example, the veil is resilient to metallic jacket cracking due to the high strength carbon core which provides some level of conductivity regardless of the mechanical strain. This is an advantage over polymer core fibers which become open-circuit under high loading conditions [8].

An example of a composite conductor veil manufactured by the authors is given in Fig. 1. Details of the plating process have been reported in [11]. The plating process produces a smooth and even copper jacket over each of the carbon fibers. Due to the inclusion of a weak binder in the veil, some small regions do not fully plate as illustrated in Fig. 1 (b). However, this has a negligible effect on the bulk conductivity of the plated veil as evident in [11].

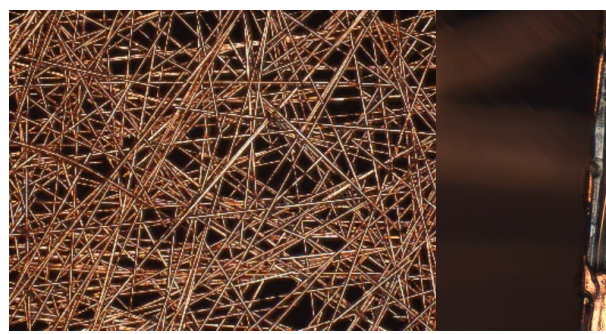

(a)

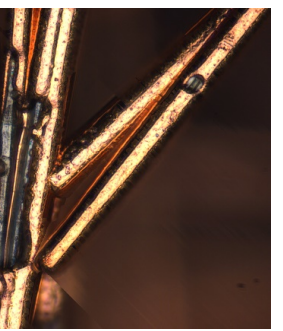

(b)

Fig. 1. (a) Example of copper plated carbon fiber veil material, (b) magnified illustration of the copper plated veil. Each carbon fiber is approximately $7.0 \mu \mathrm{m}$ in diameter.

\section{A. Laser Ablation and Transfer Methods}

The high emissivity of carbon makes it ideal for a laser patterning process. With the inclusion of a thin metallic jacket, the veil can still be cleanly cut with an infrared $\mathrm{CO}_{2}$ laser manufactured by Trotec. For the best results however, an ultraviolet laser should be used if a thick metallic jacket is required.

Although lasers provide a means for patterning the veil, they do not provide a means for transferring fine veil patterns onto composite materials. The novel solution presented here is to use a weak adhesive layer to hold the veil in position against a sacrificial backing during the laser patterning process. Since pre-preg materials are impregnated with a 'tacky' resin, they can be used to pull the desired pattern away from the sacrificial backing. This pre-preg (with carbon veil patterning) remains flexible until cured and so can be draped over a conformal shape.

After significant testing, it was found that a Teflon release film (A4000 release manufactured by Airtech) provides the perfect sacrificial backing. Bonding this release film to a metallic plate holds the patterns in position. After the laser patterning process, the waste release film can be pulled away and in doing so, provides a means for removing the waste veil cleanly. The remaining pattern can then be cleaned with air to remove any residual carbon fibers. An illustration of the removed release film showing the waste veil is illustrated in Fig. 2. The release film holding the veil is indicated by the reddish tinge. These patterns form some of the complex tests undertaken to characterize the laser profiling process. The laser power and speed settings have been varied between each of the unit cells. The darker regions indicate settings that do not provide sufficient power to cleanly cut the veil.

The bond strength between the release film and the metallic base plate is greater than the bond strength between the veil and release film. But the bond strength between the veil and prepreg materials is greater still! Hence, once the patterning process is complete, a layer of pre-preg can be transferred onto the veil pattern and easily peeled away to reveal the desired AIS.

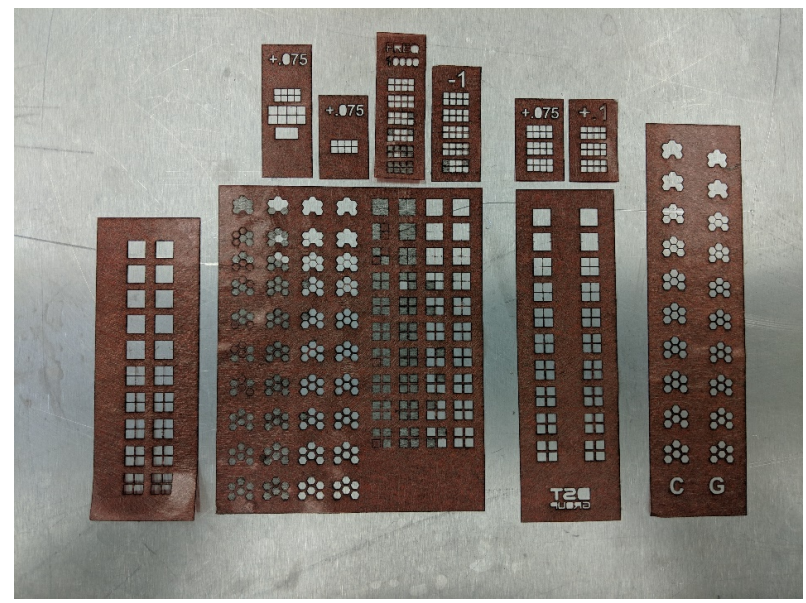

Fig. 2. Examples of release film post laser patterning.

An example of a $3.0 \mathrm{~mm} \times 3.0 \mathrm{~mm}$ AIS unit cell (consisting of a $100 \mu \mathrm{m}$ laser cut gaps) transferred onto an epoxy S-Glass pre-preg (GMS EP-280, $290 \mathrm{gsm}$ ) is illustrated in Fig. 3. This is the finest gap dimension the laser system can produce and therefore represents the hardest test case. Yet the transfer method yielded a $99.9 \%$ success rate as calculated from the 10 unit cells that failed to transfer out of the total 10,000 in Fig. 3 (a). This is important when considering the complex and largescale patterns required to realize holographic AIS.

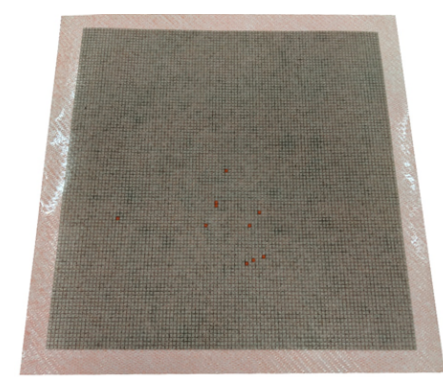

(a)

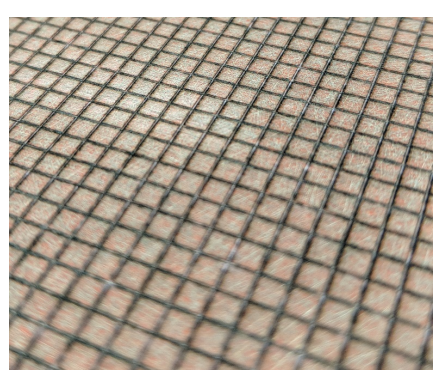

(b)
Fig. 3. (a) Linear AIS pattern of 10,000 squares and (b) close-up of unit cells measuring $3.0 \mathrm{~mm} \times 3.0 \mathrm{~mm}$ transferred directly onto GMS EP-280 pre-preg. Unit cells that failed to transfer are indicated by the remaining 'red' Teflon release film in (a).

\section{AIS DESIGN, MANUFACTURE AND RESPONSE}

\section{A. Design}

A $3.0 \mathrm{~mm} \times 3.0 \mathrm{~mm}$ unit cell (with $0.1 \mathrm{~mm}$ laser cut gaps) was chosen to exhibit a resonance within the $\mathrm{X}$-band. To test 
the precision of the laser patterning process, the band-gap response of a 5-ply EP-280 panel was analyzed using a metallic backed reflection measurement. An illustration of the ply consolidation process is illustrated in Fig. 4 (a). As the veil is a highly porous material, a key performance parameter is weather the effective impedance can be predicted sufficiently using commercial electromagnetic modeling software such as CST Microwave Studio.

The cured permittivity of the S-Glass is similar to FR-4 with $\varepsilon_{\mathrm{r}}=4.26$ and $\operatorname{Tan} \delta=0.02$ at $10.0 \mathrm{GHz}$. This was measured using the Nicholson-Ross method within a coaxial transmission line. The five plies of the EP-280 material were then consolidated to yield a panel with mean thickness of $1.375 \mathrm{~mm}$. Residual stress in the cured laminate resulted in a small air gap of approximately $0.3 \mathrm{~mm}$ between the AIS and metallic ground plane during testing. This gap was incorporated in the Floquet unit cell simulation in CST MWS 2018.

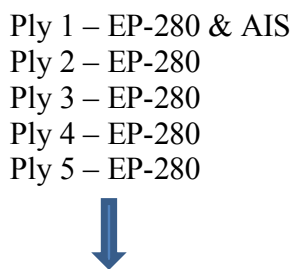

Consolidated Laminate
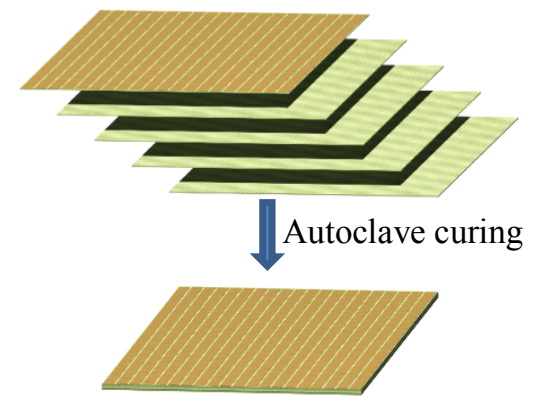

(a)
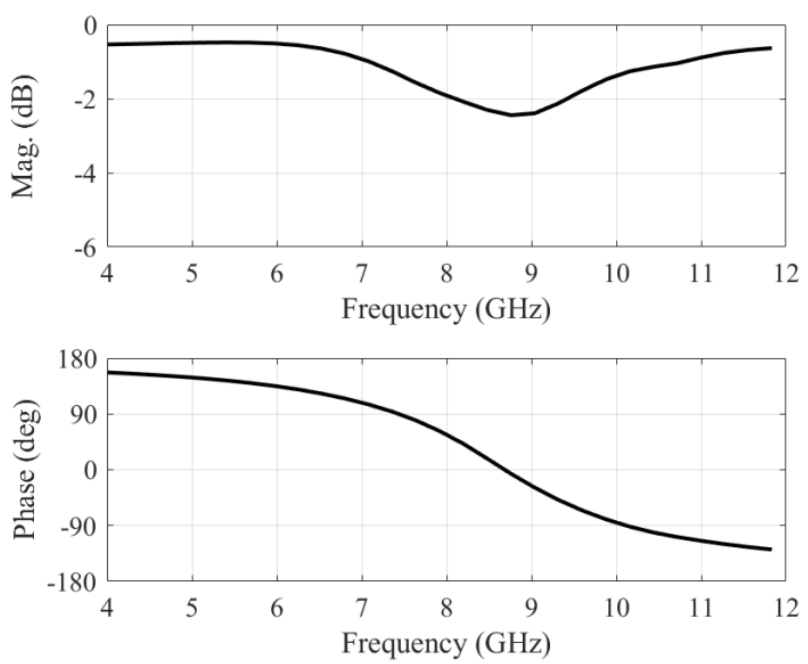

(b)

Fig. 4. (a) Illustration of the ply consolidation process and (b) simulation results of a $3.0 \mathrm{~mm} \times 3.0 \mathrm{~mm}$ AIS veil panel at normal incidence.

The designed response of the AIS panel has been provided in Fig. 4 (b). Both TE and TM polarizations are identical with a resonance centered at $8.68 \mathrm{GHz}$.

\section{B. Manufacture}

The patterned EP-280 ply was consolidated with four extra plies as illustrated in Fig. 4 (a). The material was then de-bulked under vacuum and cured in an autoclave at $120^{\circ} \mathrm{C}$ and 1 bar pressure for $3 \mathrm{hrs}$ as per the EP-280 recommended cure cycle.

As mentioned previously, the pre-preg material remains flexible until cured and can, therefore, be formed over complex conformal shapes. However, for simplicity in the characterization of the transferred pattern, a planar laminate was manufactured. The sheet consisted of 10,000 individual patches creating a total panel size of $300 \mathrm{~mm}$ x $300 \mathrm{~mm}$. The cured panel was tested in an anechoic chamber as illustrated in Fig. 5. Note that a small $10.0 \mathrm{~mm}$ thick S-Glass frame is present around the perimeter of the panel. This was assumed to have little effect on the measured specular response.

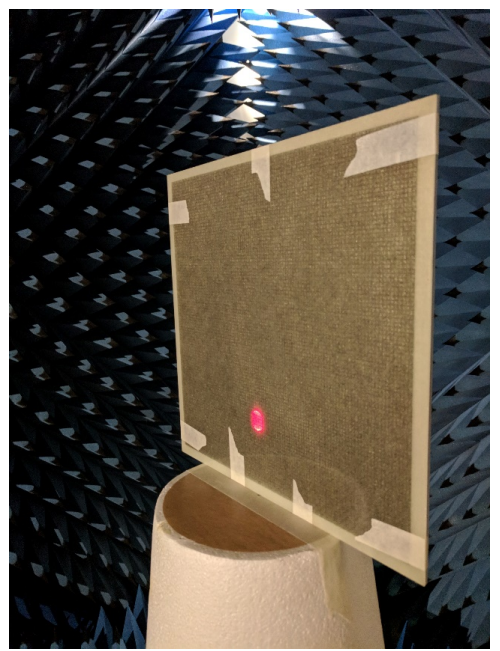

Fig. 5. Measurement of AIS patches in the DST anechoic chamber.

\section{Response}

The reflected phase and magnitude of the AIS panel was measured in an anechoic chamber relative to a $320 \mathrm{~mm} \times 320$ $\mathrm{mm}$ square metallic backing plate as illustrated in Fig. 6. Due to the finite size of the anechoic chamber, radar cross-section was not extracted since the measurements were taken in the near-field region.

The AIS exhibited a resonance (as indicated by the phase response) at $8.72 \mathrm{GHz}$ which compares favorably with the simulation results illustrated in Fig. 4 (b). It should be noted that the magnitude response of the AIS suggests there is some variation in the gap dimension. However, the bulk response compares well with the simulated result.

The good agreement between simulated and measured results for the AIS panel indicates the laser patterning process provides sufficient accuracy to develop holographic AIS. An example of a holographic AIS test pattern has been included as part of the future work for this research (see Fig. 7). The holographic patterns were transferred using the process presented herein. Further results of this holographic AIS will be presented at the conference. 

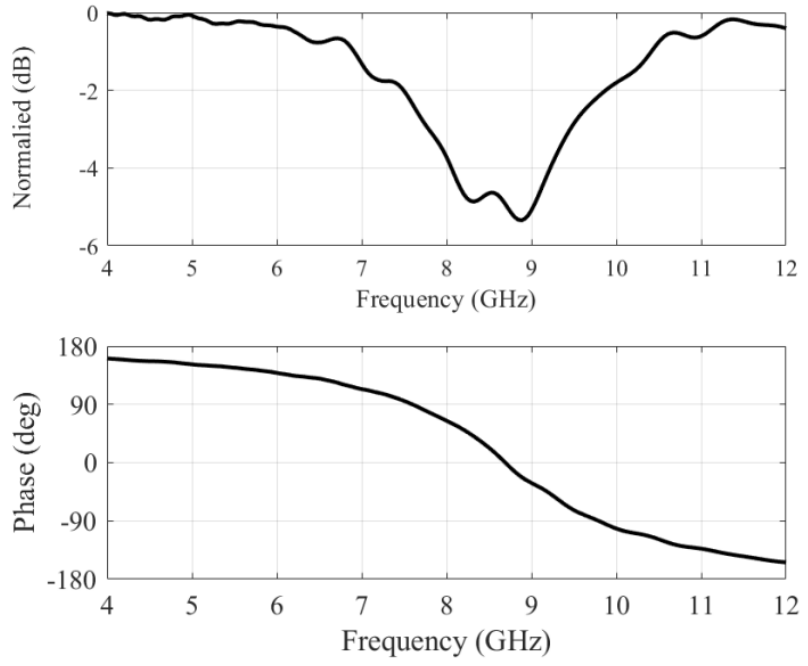

Fig. 6. Measured results of a $3 \mathrm{~mm} \times 3 \mathrm{~mm}$ AIS veil panel. (top) measured reflected magnitude at normal incidents and (bottom) reflected phase.

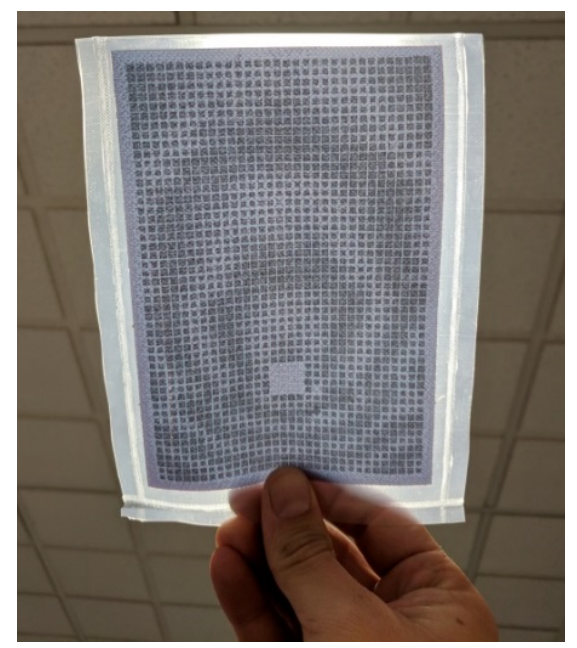

Fig. 7. An example of patterned holographic AIS on uncured pre-preg.

\section{CONCLUSION}

This paper has presented a unique carbon veil patterning process to aid in the fabrication of AIS directly on aerospace composite materials. This process can be adapted to develop conformal patterns to realize holographic AIS in complex aerospace geometries with negligible impact on the aerodynamic drag or mechanical performance of the structure. The laser ablation and transfer method was demonstrated with an accuracy of approximately $100 \mu \mathrm{m}$. The manufacturing accuracy was confirmed by the excellent agreement between simulation and the measured response for a uniform $3.0 \mathrm{~mm} \mathrm{x}$ $3.0 \mathrm{~mm}$ carbon veil AIS panel. Future work will focus on patterning with higher conductivity veils for AIS applications. This will include copper plate carbon veil as illustrated in Fig. 1. Future work will consider the development of holographic AIS with integrated active transceiver components. Hence, this work will progress the development of CLSS structures with the goal to demonstrate a complete multifunctional component on a suitable platform.

\section{ACKNOWLEDGMENT}

This work was supported by the Australian Department of Defense; Defense Science and Technology Group (DST Group) SRI-SME; the Defense Science Institute, an initiative of the State Government of Victoria

\section{REFERENCES}

[1] B. H. Fong, J. S. Colburn, J. J. Ottusch, J. L. Visher, and D. F. Sievenpiper, "Scalar and Tensor Holographic Artificial Impedance Surfaces," Antennas and Propagation, IEEE Transactions on, vol. 58, pp. 3212-3221, 2010.

[2] Y. B. Li, X. Wan, B. G. Cai, Q. Cheng, and T. J. Cui, "FrequencyControls of Electromagnetic Multi-Beam Scanning by Metasurfaces," Scientific Reports, vol. 4, p. 6921, 11/05/online 2014.

[3] F. Caminita, E. Martini, G. Minatti, and S. Maci, "Fast integral equation method for metasurface antennas," in URSI International Symposium on Electromagnetic Theory (EMTS), 2016, pp. 480-483.

[4] G. Minatti, F. Caminita, E. Martini, and S. Maci, "A Floquet Wave theory for curvilinear locally periodic boundary conditions," in URSI International Symposium on Electromagnetic Theory (EMTS), 2016, pp. $565-568$.

[5] F. Silvestri, A. Benini, E. Gandini, G. Gerini, E. Martini, S. Maci, et al., "DragOnFly - Electronically steerable low drag aeronautical antenna," in 11th European Conference on Antennas and Propagation (EUCAP), 2017, pp. 3423-3427.

[6] P. J. Callus, "Novel concepts for conformal load-bearing antenna structure," Defence Science and Technology Organisation (DSTO) 2008.

[7] K. Nicholson, W. Rowe, K. Ghorbani, and P. Callus, "Split-ring resonator loaded miniaturized slot for the Slotted Waveguide Antenna Stiffened Structure," presented at the Fifth International Congress on Advanced Electromagnetic Materials in Microwaves and Optics, 2011.

[8] K. Nicholson, O. Dunbabin, T. Baum, and K. Ghorbani, "Characterisation of integrated microstrip lines in aerospace composite structure", Electronics Letters, vol. 53, no. 1, pp. 36-38, 2016

[9] T. C. Baum, R. W. Ziolkowski, K. Ghorbani, and K. J. Nicholson, "Investigations of a Load-Bearing Composite Electrically Small Egyptian Axe Dipole Antenna," IEEE Transactions on Antennas and Propagation, vol. 65, pp. 3827-3837, 2017.

[10] T. C. Baum, R. W. Ziolkowski, K. Ghorbani, and K. J. Nicholson, "Embroidered Active Microwave Composite Preimpregnated Electronics - Pregtronics," IEEE Transactions on Microwave Theory and Techniques, vol. 64, pp. 3175-3186, 2016.

[11] M. Rudd, T. C. Baum, B. Mapleback, K. Ghorbani, and K. J. Nicholson, "Reducing the Attenuation in CFRP Waveguide Using Carbon Fiber Veil," IEEE Microwave and Wireless Components Letters, vol. 27, pp. 1089-1091, 2017.

[12] L. Li, T. Jun Cui, W. Ji, S. Liu, J. Ding, X. Wan, et al., "Electromagnetic reprogrammable coding-metasurface holograms," Nature Communications, vol. 8, p. 197, 2017/08/04 2017.

[13] A. Bojovschi, K. J. Nicholson, A. Galehdar, P. J. Callus, and K. Ghorbani, "The role of fibre orientation on the electromagnetic performance of waveguides manufactured from carbon fibre reinforced plastic," Progress In Electromagnetics Research B, vol. 39, pp. 267-280, 2012.

[14] D. J. Gregoire, "3-D Conformal Metasurfaces," IEEE Antennas and Wireless Propagation Letters, vol. 12, pp. 233-236, 2013.

[15] D. Sievenpiper, J. Colburn, B. Fong, J. Ottusch, and J. Visher, "Holographic artificial impedance surfaces for conformal antennas," in Antennas and Propagation Society International Symposium, 2005 IEEE, 2005, pp. 256-259 vol. 1B. 